UC-20

Repurting Date: October 1974 Issued: November 1974

\title{
Potential Fatigue Problems in First-Wall Laser-Controlled Fusion Reactors
}

by

\section{J. Woortman*}

"LASL Consultant. Permanent address: Department of Matorials Science, Northwestern University, Evaneton, IL 60201.

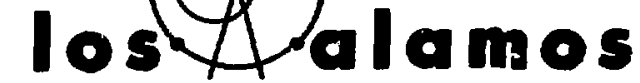

scientifte laboratory

of the University of California

LOS ALAMOS, NEW MEXICO 87544

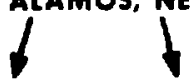


In the interest of prompt distribution, this LAMS report was not edited by the Technical Information staff.

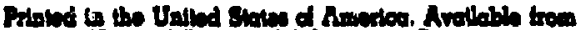

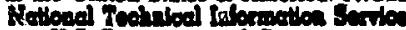

U.3. Depoptact of Comerto

5 Fot logh pod

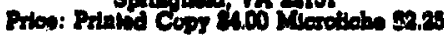

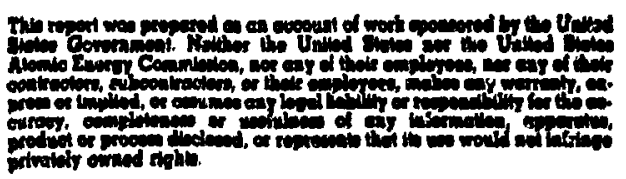


POTENTIAL PATIGUE PROBLEKS IN FIRST.

WALL LASER-CONTROLLED FUSION REACTORS

by

J. Weertwan

\section{ABSTRACT}

In this report an eativate is ande of posalble fatigue problens that are likely to be encountered in the metal firat wall of laber-co!,trolled thermonuclear (fuston) reactors (LCTRs) A reviev is given of different sources that produce fluctuating stresses. The iargest strasses occur in the farm of short wovelength, thersoelastic stress pulses that start at the Inner wall and wove into the Inter lor of the netal shell. It is concluded that it is Iikely (as suggested by zukas )that these short wavelength stress pulses ate atcenunted rapidly with distance. If they are Indeed attenuated otrongly, high aplitude stress pulses ahould not cause fat1gue fallure of firet wall wetal shells. (Other sources produce cyclic streases that are of too low an anplitude to cause fatigue fallure.) Measuresence are needed of the internal friction of possible first wall ater1als taken under conditions of high gtress aplitude, and ratietion danage will Indicate which aeterials can attenunte raptdly high aplitude, short wavelength streas pulees and thus be resistant to fatigue fallure. Experisents also are needed to deterwine if fatigue crack growth can be produced by 14-HeV neutron collision cascades. Whether such crack growth can occur is only a theoretical specuiation at the present tine. If It does occur after a year's operation firot-wall aterial may contain a high density of itlilinetar length cracke. (Stresses are calculated for sphexical niobiu chell of radius 2 and thickness $1 \mathrm{cs}$. These stresees generally can be converted to the values appropriate to a ntobium shell of radius $R$ by aultiplying by the factor $(R / R)^{2}$, where $R=2$. Since the elastic modull of other metale considerid for a firar wall (olybjenum, vanadiun, stainless sieel) are of the sare crder as the modulus

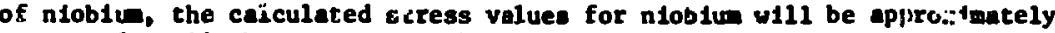
correct for all these aterials.)

\section{INTRODUCTION}

In proposed designs ${ }^{1-7}$ of laser controlled sinereoriuclear reuctore (LCTRB) deuter1um-trit1us micropellets are fusion burneỏ at a rate between 1 and $10 \mathrm{~Hz}$. Each pallet produces about $100 \mathrm{~kJ}$ of energy in 1to explosive bum. It Is inevitable that the asterial out of which an LCTR is constructed will be ubjected to fluctuating streases because of these perlodic burste of fualon -crented energy. Since fluctuating otresses can cause fatigue fallure of material, potential fatigue probleas any exist for LCTR. It 1s the purpose of this inforal report to exanine the potential fatigue problens of tha first- wall anterial of LCTRs. A review is glven firat of the nources of the fluctuating stresses and the estated magnitude of these stresses. Th1s review is followed by an eatiate of the serlousness of the fatigue dange 11kely to be suffared by the firstwall waterial.

\section{It. SOURCES OF FUCTUATIHG STRESSES}

An eatinaced energy release spectrum from a 99MJ microexplosion of a DT pellet (taken frow Refs. 1 and 5) is given in Table 1 . Suppose that, periodIcally, DT pellets are fugion burned at the center 
TABLE I

TYPICAL ENERGY-RELEASE MECHAIENS EROH A 99-HJ DT PELLET MCROEXPLOSION ${ }^{2}, 5$

Fraction of Total

Mechanie

\section{$X$ rays}

Apha Farticles that escope plasen

Plaona kinecte ensrzy

alpha particleo

deuterong

tritone

Heutrots
Bneray pelanes
0.01
0.07
0. 15
0.73

\section{Patelcles per Bulue Avarare Eneroy per Parefele}

$2.2 \times 10^{19}$

- 4 kav peak

$1.3 \times 10^{19}$
$1.2 \times 11^{20}$
$1.2 \times 10^{20}$

$0.6 \mathrm{HeV}$

$0.3 \mathrm{KeV}$ total average $0.37 \mathrm{HeV}$

0.4 MeV of a spher tcal first wall wecal shell of radiug $R$ and thickness $\Delta R$. thet cyclic streses are produced within the atal shell by aicroexplosions of the pulleca?

A. Stregs Bulees Produced by Konencue Tranter An upper linte can be sec to the asentcuda of: the strese pulise produced by the transfer to the she11 of the monettun of the neucrons produced in a DT pellet burn if it is assurted that all the natrons are absorbed uniformly within the eatel shal. (Accualiy aost of the neutrone w111 be absorbed in the Iiquid Lithiu blanket that surrounde the firat wil) In an incresental soldd anglo $6 \Omega$ the monentun ca:ried by the neutrons is equal co $x_{n} n_{n} y_{n} \delta / 4 n$ where $N_{n}$ is cotal nuber of neutrons crented in a acroexplosion, 19 the aas of a neutron $\left(a_{n}-1.7 \mathrm{x}\right.$ $10^{-27} \mathrm{~kg}$ ), and $v_{n}$ la the veluclty of a neutron. The neutrons are produced in a tiee incerval (c 20 po) that is short copared with the ctre required for a gound wave to eraverese the ehtcknegs of the metal whell $\left(\sim \Delta R(0 / \mu)^{1 / 2}\right.$ - $S$ Hs for $\Delta R=1$ ca where $o$ 1s the denatty of the anterial of the shell and $\omega$ is an appropriate elastic constant). Imediately after the neutcong are absorbed in the wetal a segwent of solid angle $\delta \Omega$ of the shell has a tocal yoaencun gtven by po $R^{2} \Delta R \mathrm{~V}$, where $\mathrm{s}$ is the radial velocity of the ahell. It Is agsuned that $\Delta R$ <i R. By conservation of momentum

$$
v=\frac{N_{n} n^{v}}{4 \pi \rho R^{2} \Delta R}
$$

As the wetel shell expende it becones heressed elastically and the radtal veloctey decreares. The thell is wet into a ringing aotion. In dynanic elastictity probleas the relacionahip $(1 / 2) \mathrm{w}^{2}$. (1/2) $\sigma^{2} / \mu$ holds for any volue elenent lectust: of the principle of conservation of enargy. Here $v$ is the aximu veloctty of the volume deant, a is the strent aplitude to which the volue elenent: is gubjected, and is it an appopriare elests consent.

Thue the axime ecrees that can be produced by the trancer of 11 the momentu of the poutrons to the ahell is of the order of

$$
0=\frac{N_{n} n_{n}(\omega / D)^{1 / 2}}{4 \pi R^{2} \Delta R} .
$$

For shell wude out of cloblut $\left(D=8.6 \times 10^{3}\right.$ $\mathrm{k}_{\mathrm{B}}=-3$ and $\left.i=100 \mathrm{~cm}^{-2}\right)$ of radius $\mathrm{R}-2 \mathrm{z}$ and thicknese $R$ - 2 a the screas given by E. (2) Cor $N_{n}=3.3 \times 10^{19}$ neutrone per pellet of 14-xyy enerny

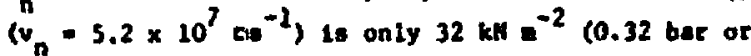
4.5 ps1). a atress aplitude uf triviel agnizude. The aipha particles that atrike tho inner wall of the shell w111 penctrate a distance d hat is wall compared with tho thell thicknase (d) -2 lat for 2uet alpha particlee in nlobluab. The cranufer of zomentutu of the alphe particlea will accur in a thickness of ancerisl of order of $d_{a}$. A stress wave wil be set ap. The atress aplitude of this uave will be of the order of

$$
\sigma=\frac{N_{a} a_{n}(u / \rho)^{1 / 2}}{4 \pi R^{2} d_{\alpha}}
$$


whire ${ }_{\alpha}$ is the number at alpha pareicles per pellek that penetrace the firat vall, a is the mase of an alpha particle $\left(a_{\mathrm{b}}=4 \mathrm{an}_{n}\right)$, and $v_{0}$ Is this velectey of the alphu percicles $\left(v_{n}=9.8 \times 10^{6} \mathrm{~m}^{-1}\right.$ for $2-\mathrm{k}, \mathrm{v}$ ulpha partleles). For $*-2.2 \times 10^{19}$ purticles per pellet, the screas glven by eq. (3) in o $-80 \mathrm{ma} \mathrm{a}^{-2}$ (80C barg of $12,000 \mathrm{pot}-12 \mathrm{ks}$ ).

Tije wavelength of the serers vavis is of the ordur of $d^{*}$. The arpll ude of the strens vave ual decrease as It eoves clirought the etal sthell because of onergy lusses caused by ineranal friction (damp (ng). If the logarichail docreserit is sepresented by $\delta$, tho serens anplicude wisl equal o fap $(-x b / d a)$ where $x$ is the destance rraveled by tho sivess wave and a ls gaven by Ea. (3). For the rattor liece vatse for the ducteans of $\&$ - 0.1 , the serest wave yould die out in a digtance of thu order $6.12=$ Fo: log dke. of $E=10^{-3}$, unty moderaraly larce yalut for she internit friction, thit stcess apilcude would not decrease appreciably in craytrains * thell of chlckncss of 2 cte.

The $x$-ray phecons of t-kev encegy mould penceraze che sheld co a diskance dy of the order of

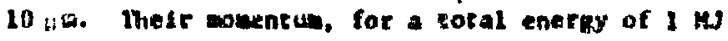
per pellet, would produce a streth pulve of enly about $1.2 \times 10^{4} \mathrm{H} \mathrm{z}^{-2}(0.12$ bar or $4.5 \mathrm{put})$.

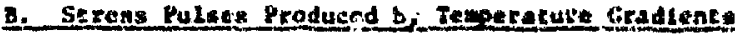

The energy depostend by the neutzons, atpho partieley, and pitatons ralsen the ceaperature of the

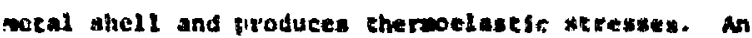
exact analyeteal solucton fot chls promies st beins developed by kay Axtord. 9 Ha haleutaced the

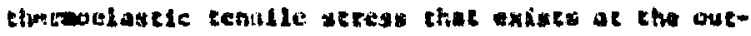
er surface of the shell Imedtacely arter atcroexplosion has caken place. His rewults tor four wata art given th Table $t h$. The colculatiche are

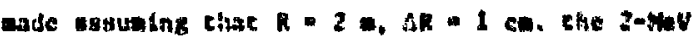
alphs particleg and the photons cegceider depwate w of enezay at the snner surface of the shell. and 4 wh of tho $7 \mathrm{~m}$ of the colal wherey of the neverons

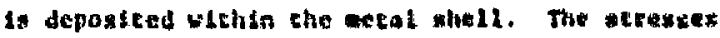
are calculated for the cane in mith intremal offest*

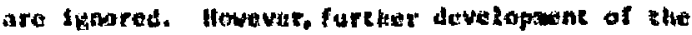

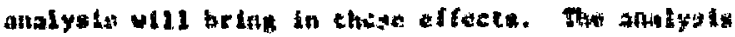

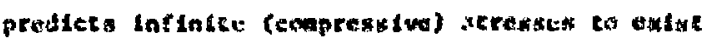
Luicially ar the Inner surface of tha shoz: becalats

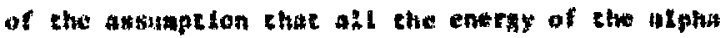

TALE 12

THESHEQASTIC STRESS AT OUTER SUKFACE of senexiche sktht

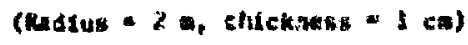

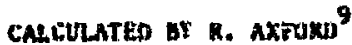

ndoblus
seatnlent steol
colyldems:
vandiup.

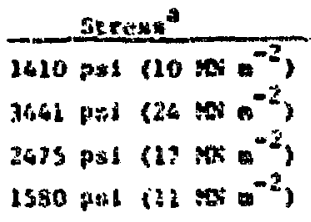

a It the enerky of clate cavicy gas In addrd to the

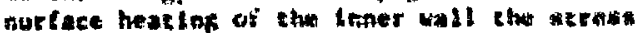
ievel. is upproximacely dowbites.

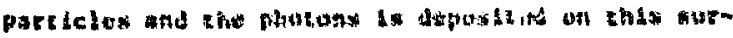

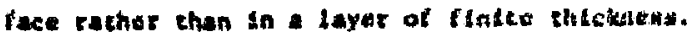

An oscteney san tat eade of the offect of tho

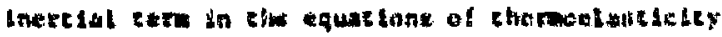

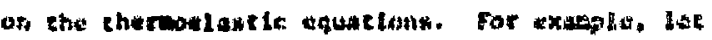

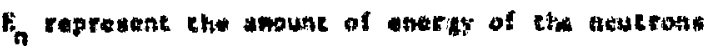

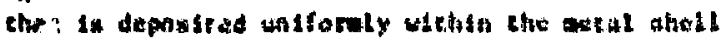

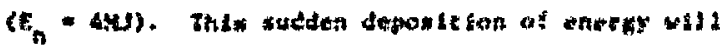

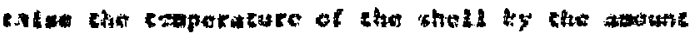
St sivan by

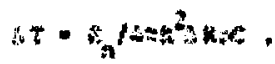

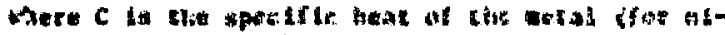

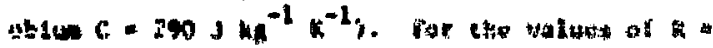

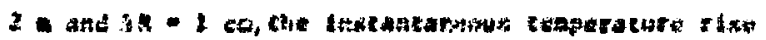

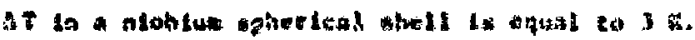

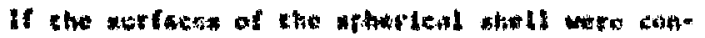

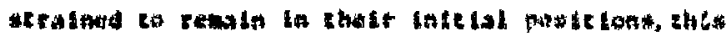

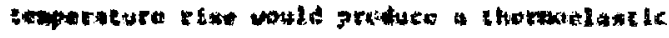

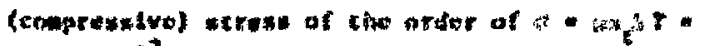

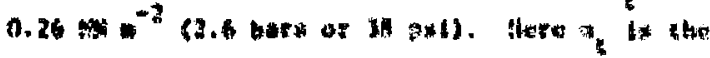

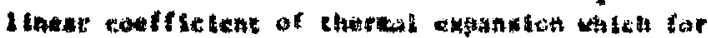

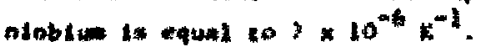

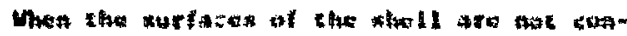

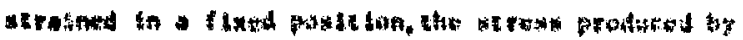

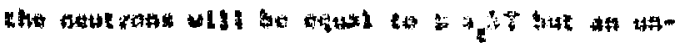

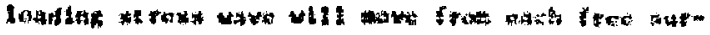

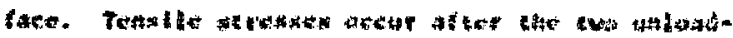

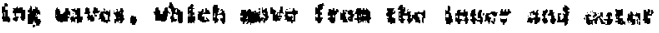




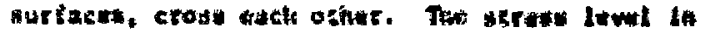

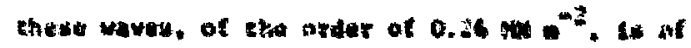
crivial zagaszute.

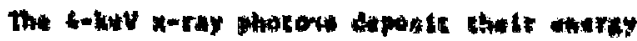

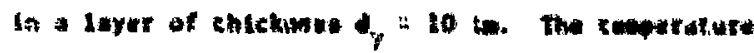

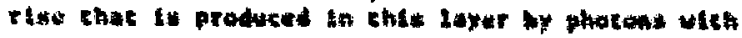
a cosell warat is

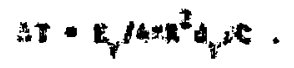

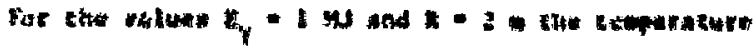

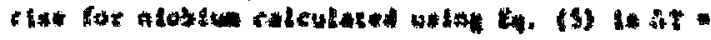

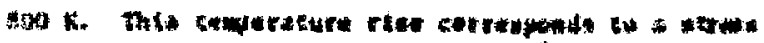

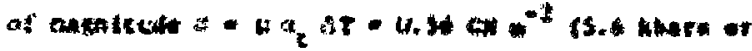

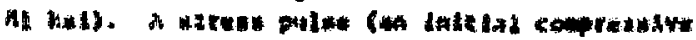

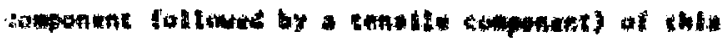

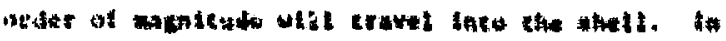

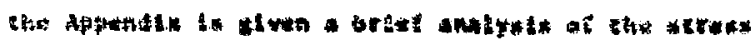

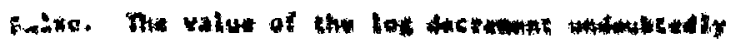

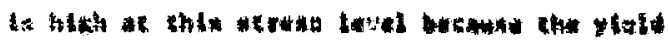

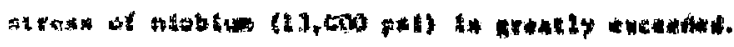

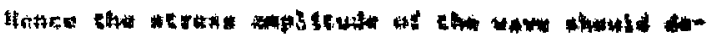

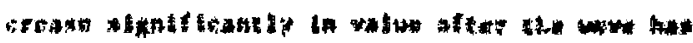

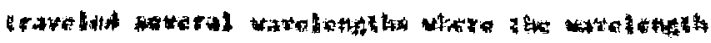

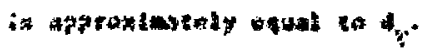

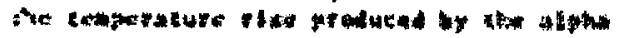

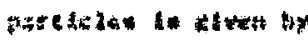

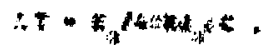

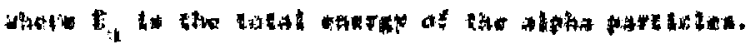

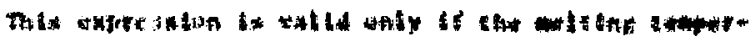

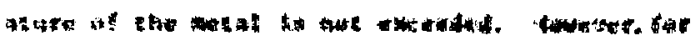

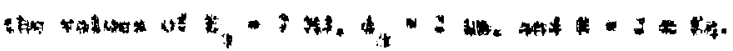

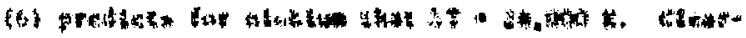

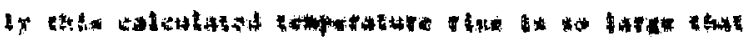

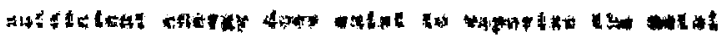

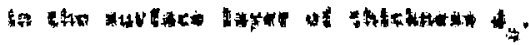

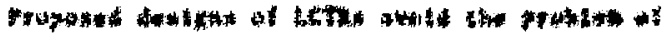

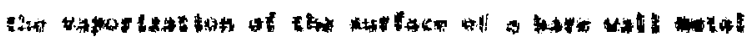

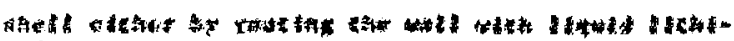

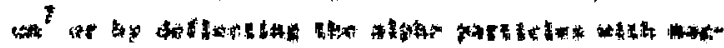
Axol

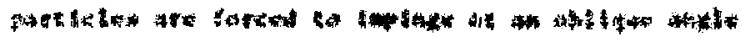

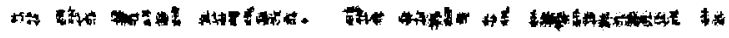

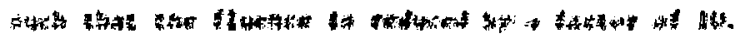

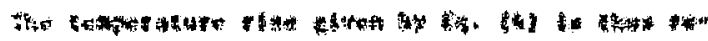

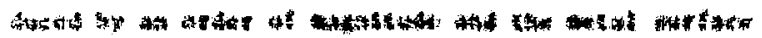

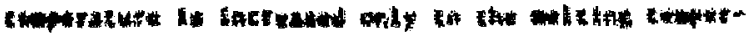

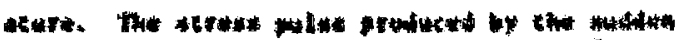

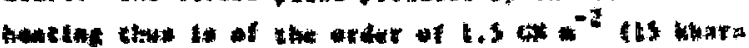

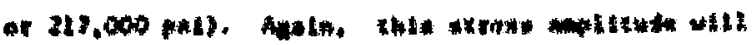

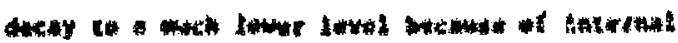

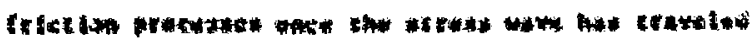

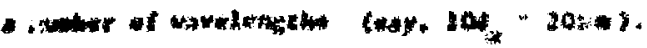

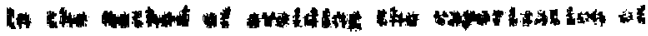

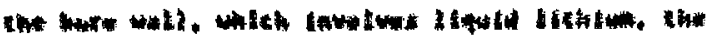

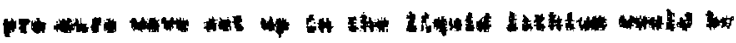

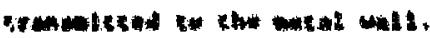

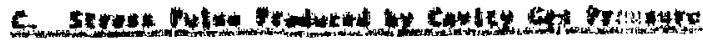

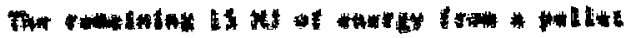

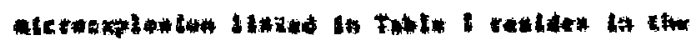

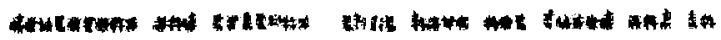

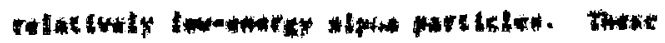

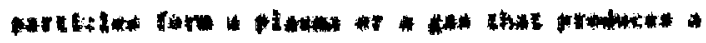

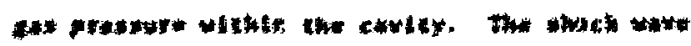

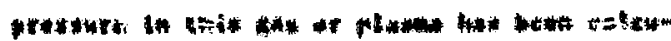

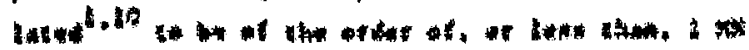

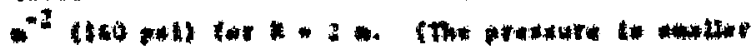

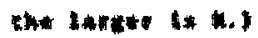

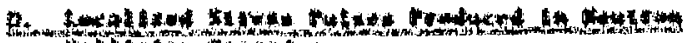

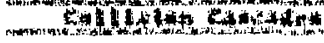

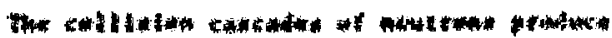

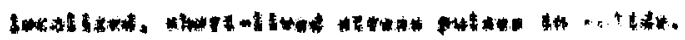

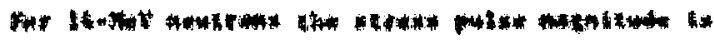

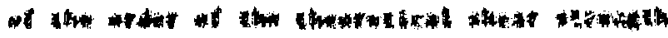

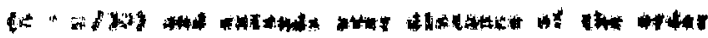

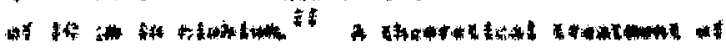

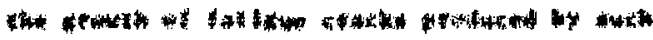

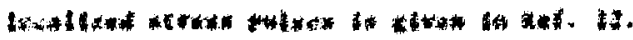

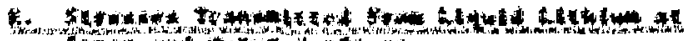
sis

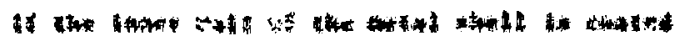
4f

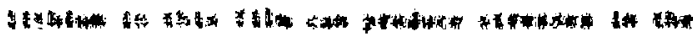

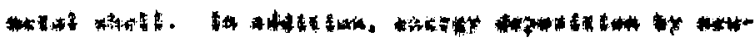

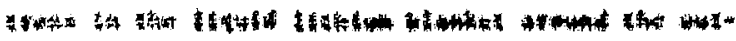

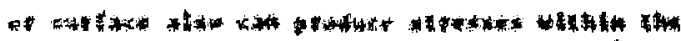
-

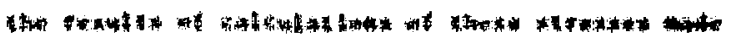

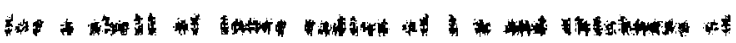
f:

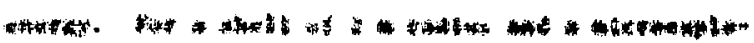

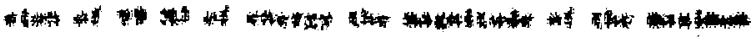

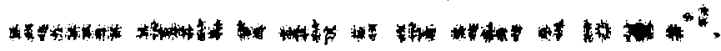


1.4kt:

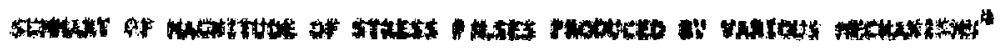

\begin{tabular}{|c|c|c|c|}
\hline 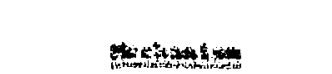 & 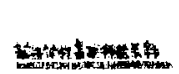 & strmit & 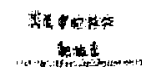 \\
\hline \multicolumn{4}{|l|}{ 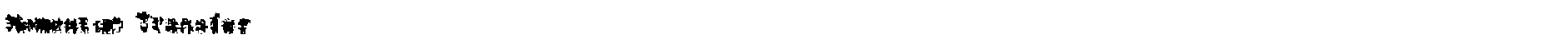 } \\
\hline 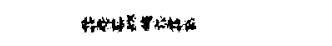 & $\because 1$ & 4.t5 & 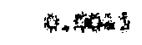 \\
\hline Alfthe & 2 & 4 & : \\
\hline 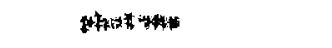 & 50 & $\$ .012$ & a. \\
\hline 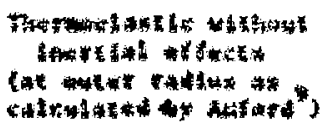 & & $\mathbf{n}$ & 1.5 \\
\hline \multicolumn{4}{|l|}{ 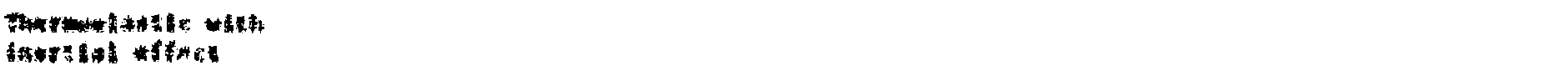 } \\
\hline 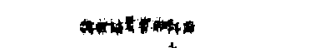 & $\theta \leqslant$ & a.3 & 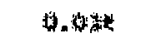 \\
\hline$A \mid t=0$ & 3 & $1+00$ & 50 \\
\hline that & 10 & $\$ 0$ & 楮 \\
\hline 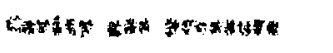 & & $\$$ & s, $1:$ \\
\hline 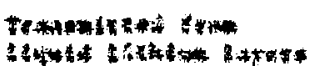 & & $\$ 0$ & $\$$ \\
\hline 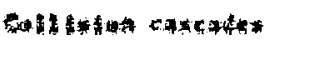 & 0.1 .6 & 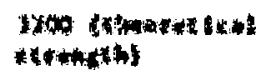 & $A+\infty$ \\
\hline
\end{tabular}

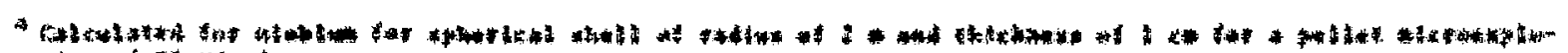

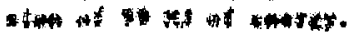

多

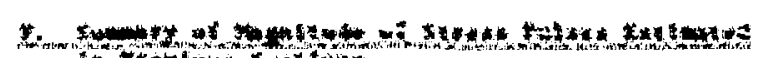
Fon

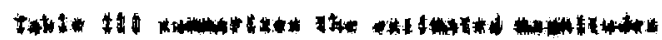

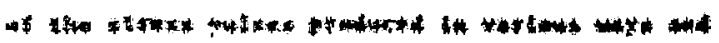

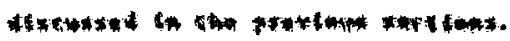

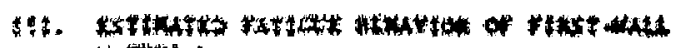

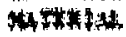

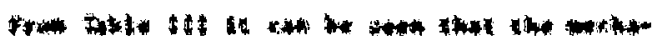

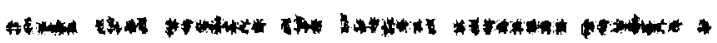

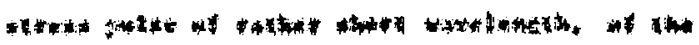

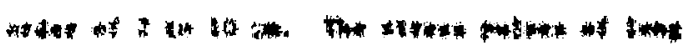

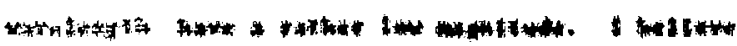

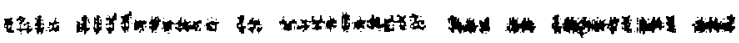

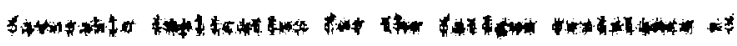
1570

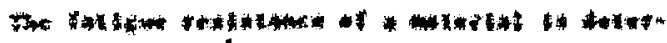

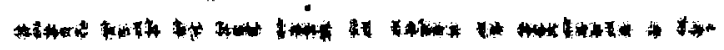

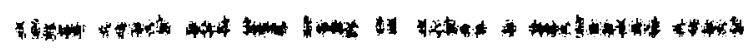

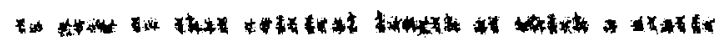

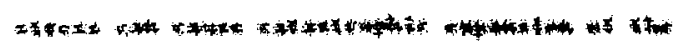

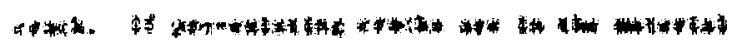

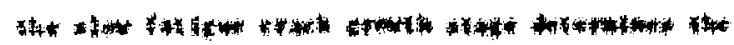

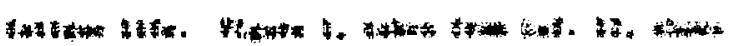

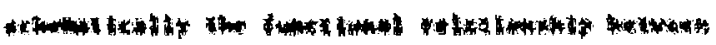

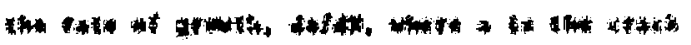
4int

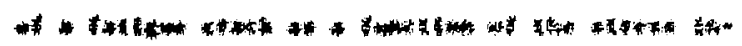

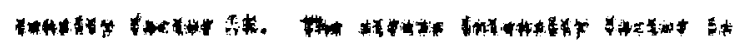

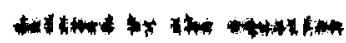

$$
x+\cdots+4
$$

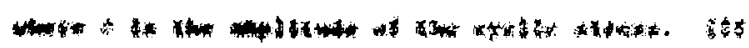

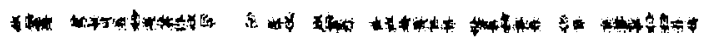

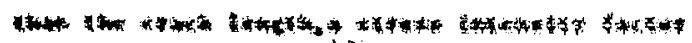

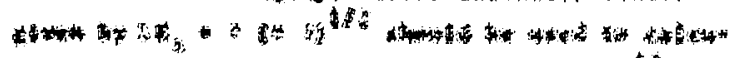

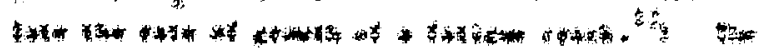

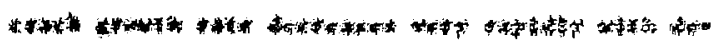

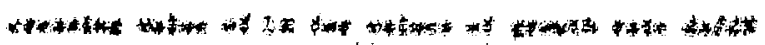

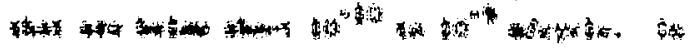
ont 


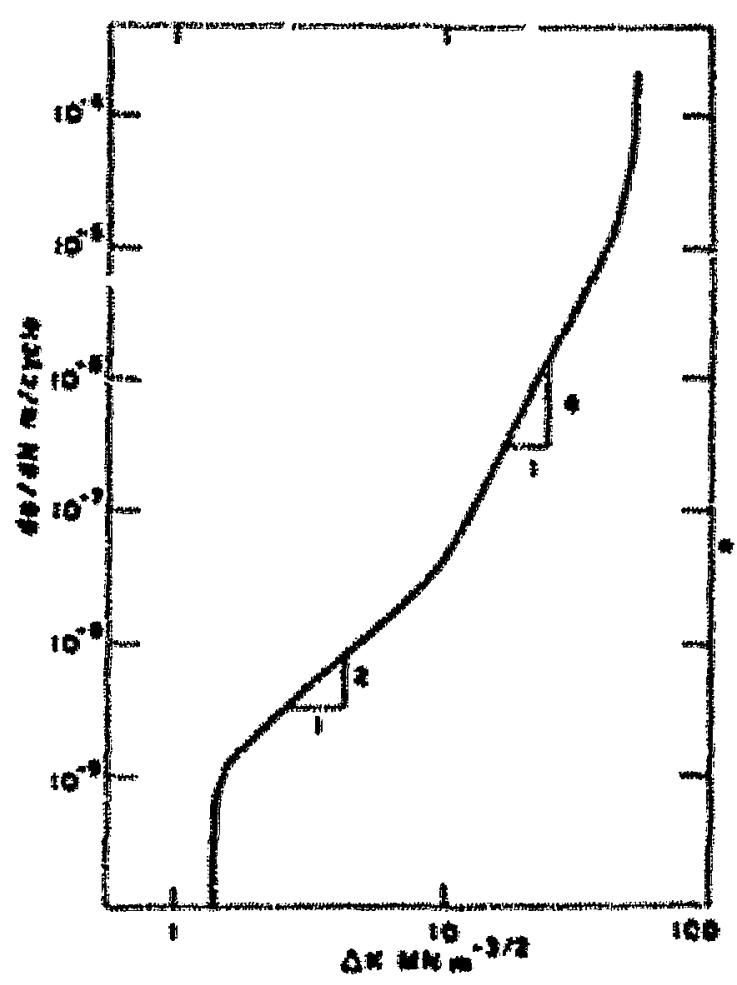

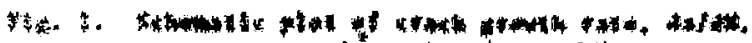

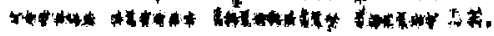

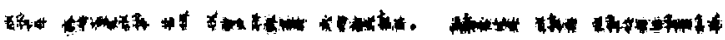

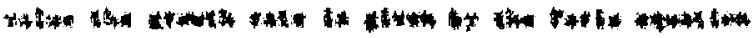

$$
40600404
$$

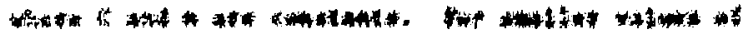
A2

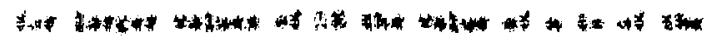

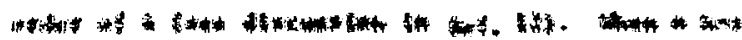

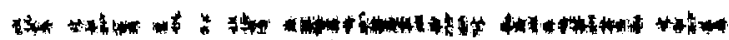

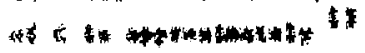

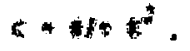

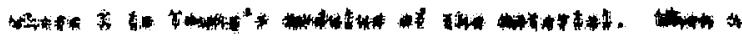

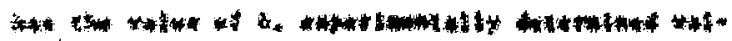

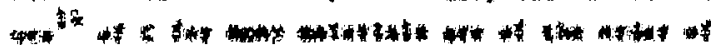
$+10^{-2} t^{-2}$

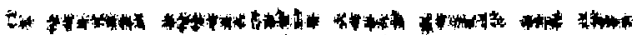

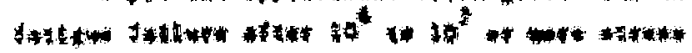

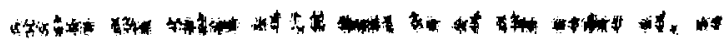

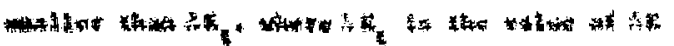

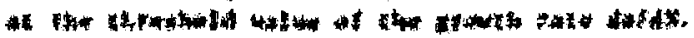

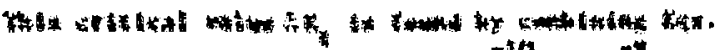

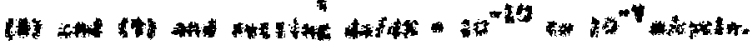
isim

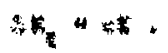

Hotro tal a

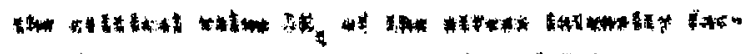

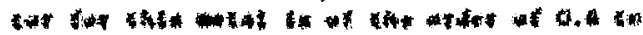

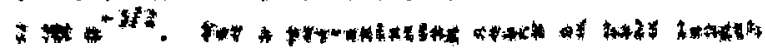

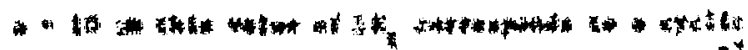

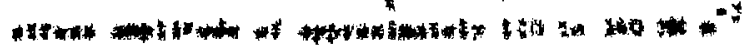

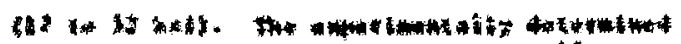

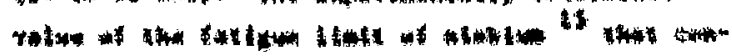

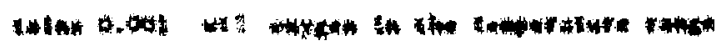

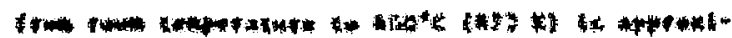

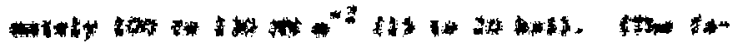

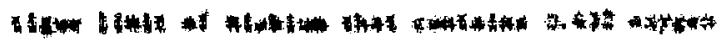

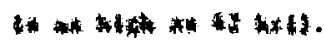

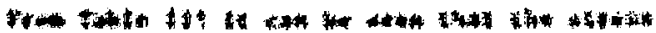

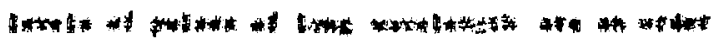

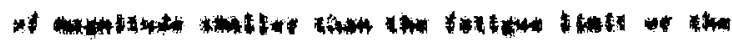

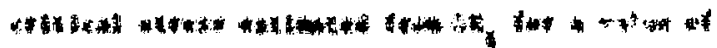

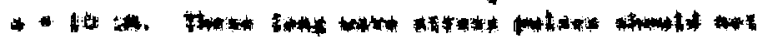

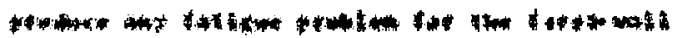

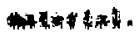

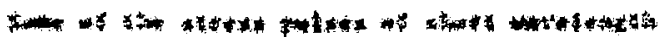

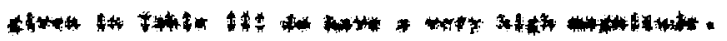

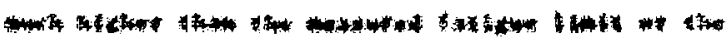

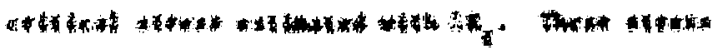

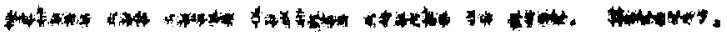

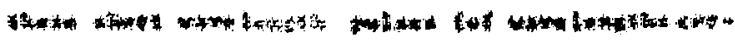

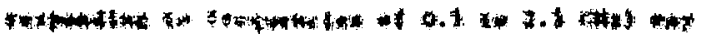

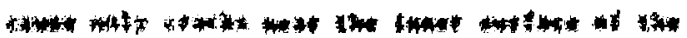

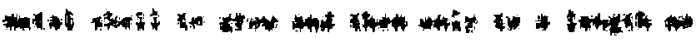

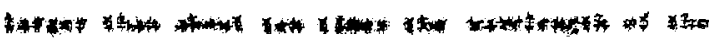

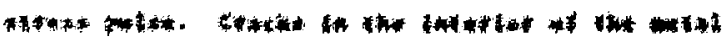

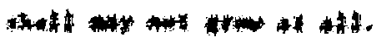

7. How

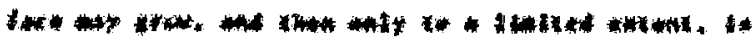

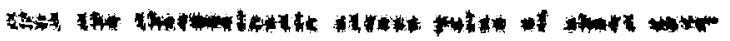

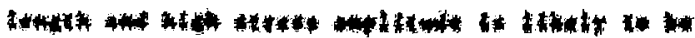

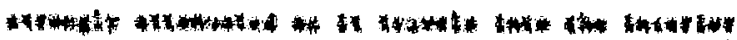

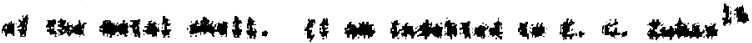
bo 


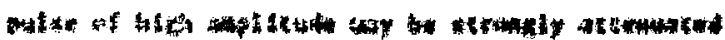
ald

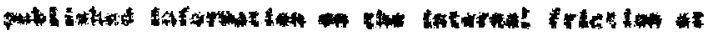

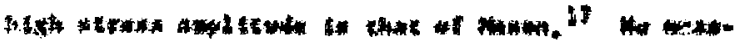

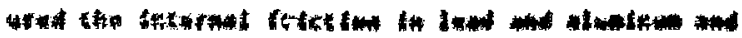

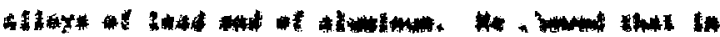

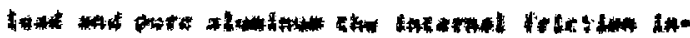

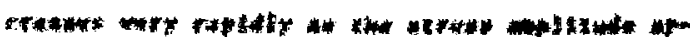

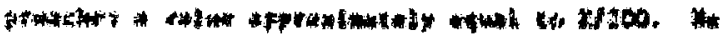
F

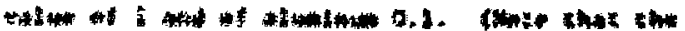

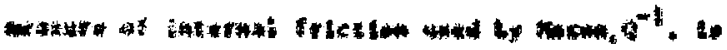
-

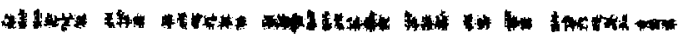

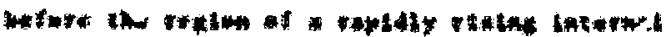

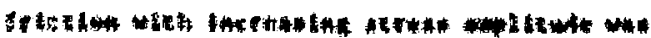

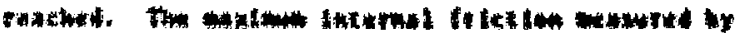

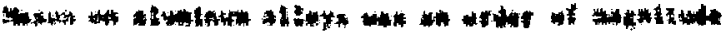

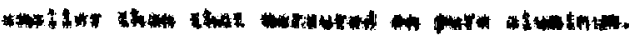

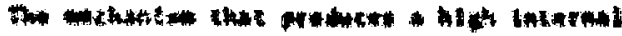

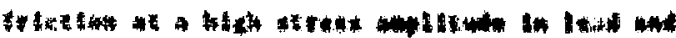

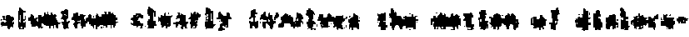
F f

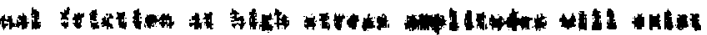

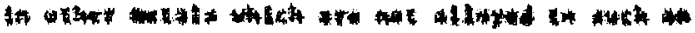

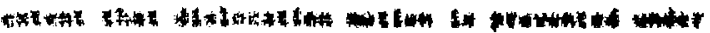

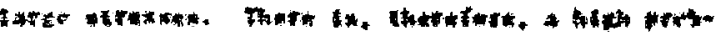

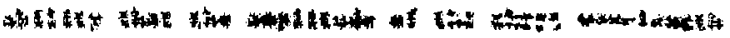

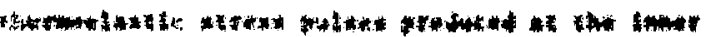

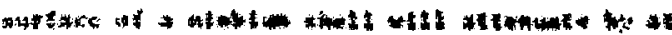

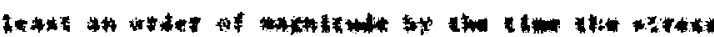

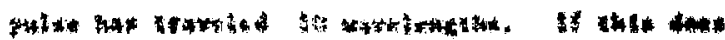

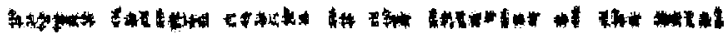

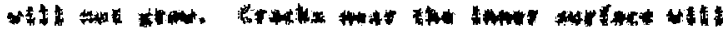

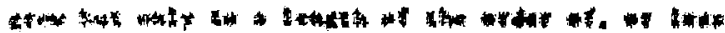

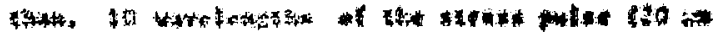

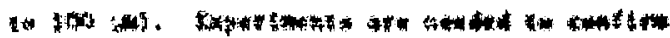

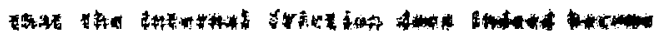

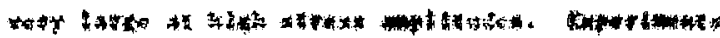
14hat

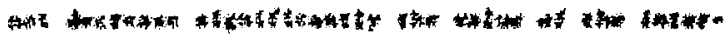

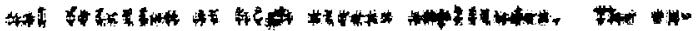

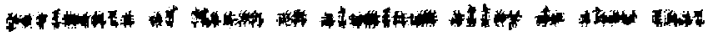

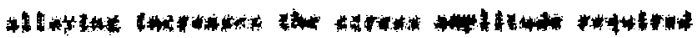

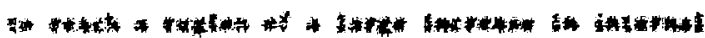

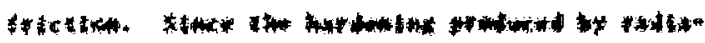

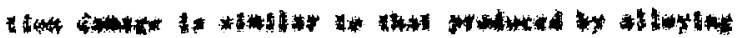

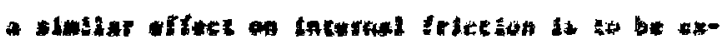
owectit.

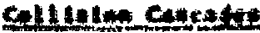

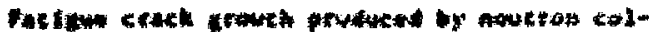

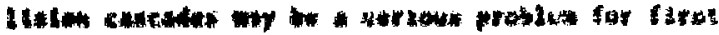

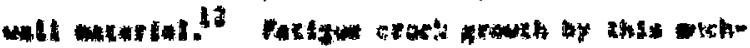

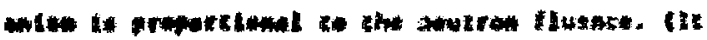

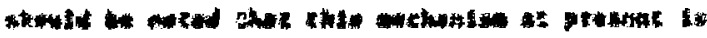

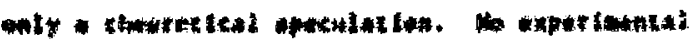

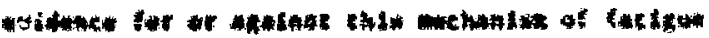

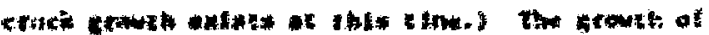

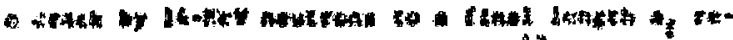

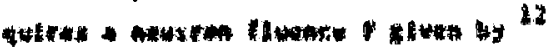

$$
+\ldots
$$

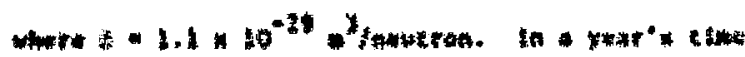

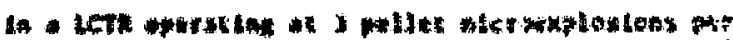

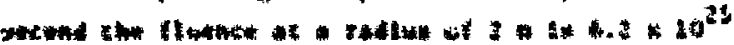

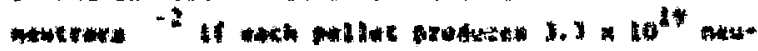

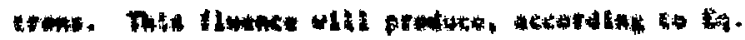

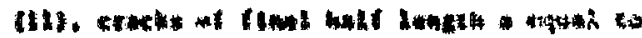

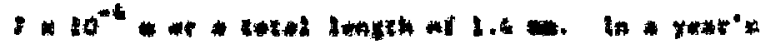

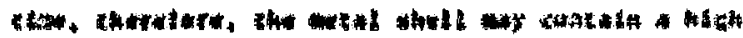

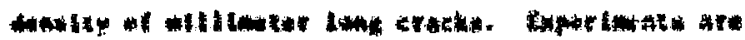

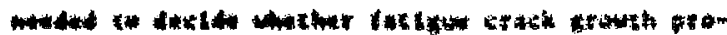
4wot hy collim

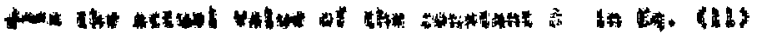

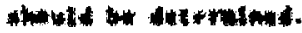

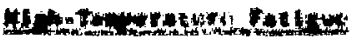

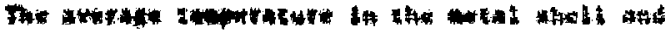

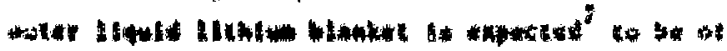

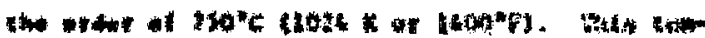

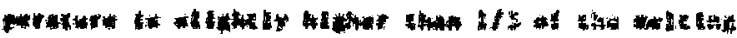

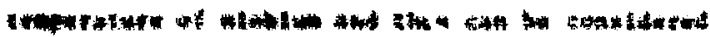

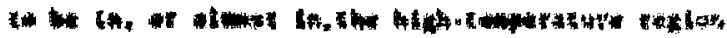

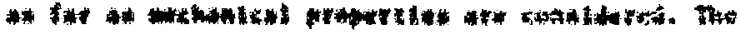

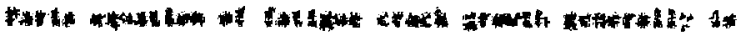

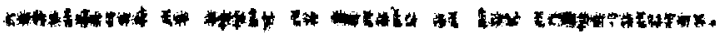

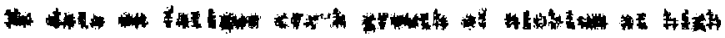

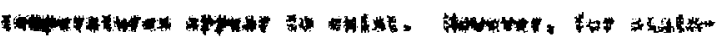

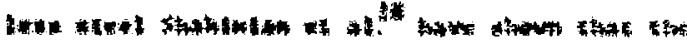

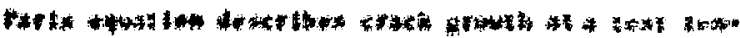

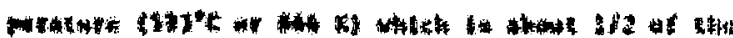

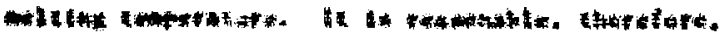

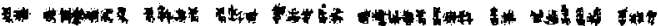




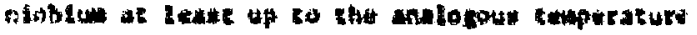
(t)

If ethe cenpuracure in relatively htob other

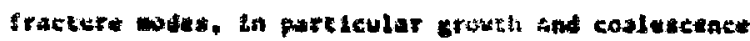

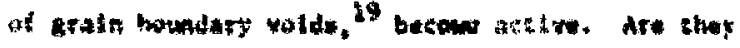

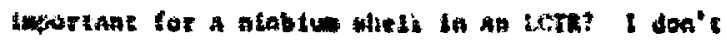

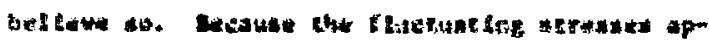
plles to the theds wet of wheh share furselon, caz-

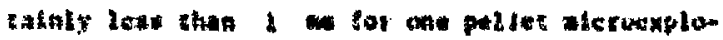

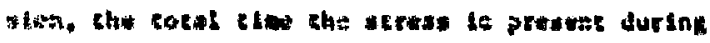

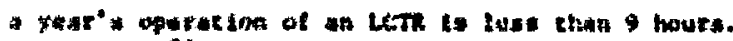

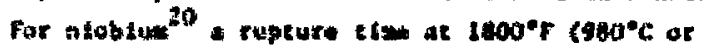

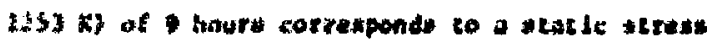

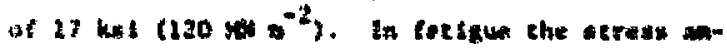

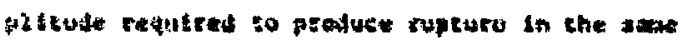

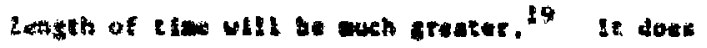

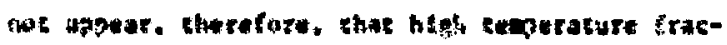

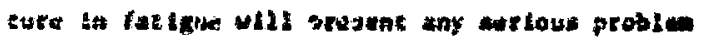

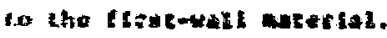

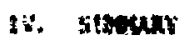

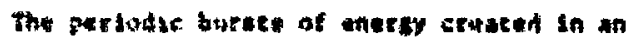

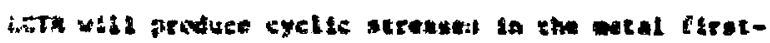

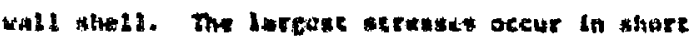

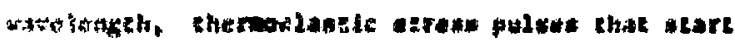

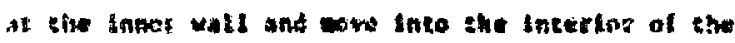

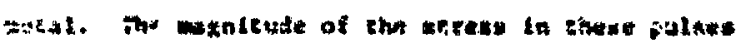

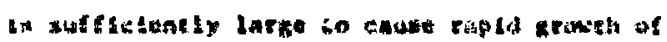

fasisum erack: and eonwequenty early fallure by fa-

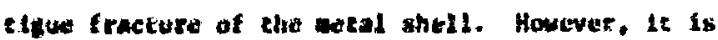
Lkely ghat thetw siore waveletath sereas pulses are actanused rapidly with distance cravelos by

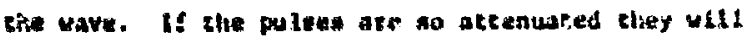
not prestine a socian fat igue problew to the wetal stell.

It In stroncly retomonded the lnterzas irtcclon mururemenes be madt at a high nertsis iapiscuds of postible first-vall ancerial to find sudtable watertals or coatingts that do tave htgh dopting properchen. internel fricilon experimenta alko chould to currticd our. undar rafteclo: dange com-

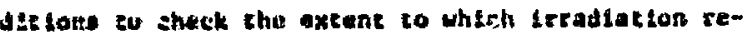
dure* the Internal friction of etwe axerlol.

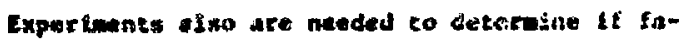
cifue ertek groien ean be produced by $14-\mathrm{keV}$ neutron zolliston cazcades. At the pranent the the poss $1-$ biliky of faclete crack erouth produced by thls cechanfere it only a chaoretteal speculatiou. If neutson costision cascader can caun crack arouth,

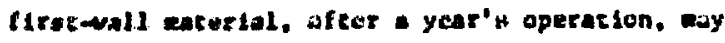
coneatn a very high censicy ot allimeter long craekx. Furthe groweh of these eracks will occur

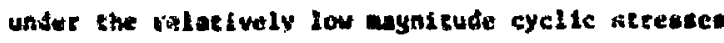
produced by the echandas: liaced in Table $l ! 1$.

Nowatx

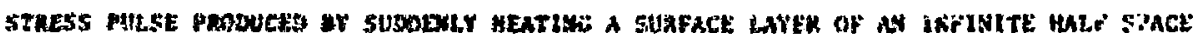

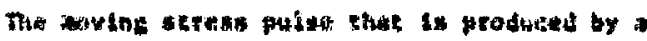

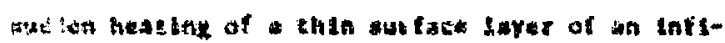

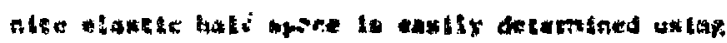

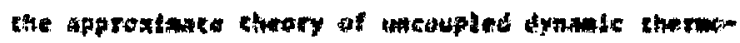

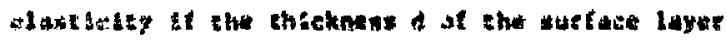

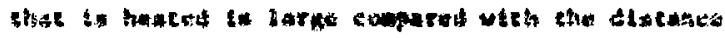

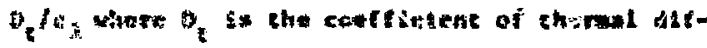

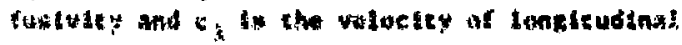

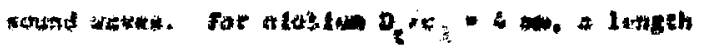

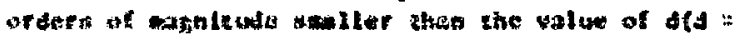

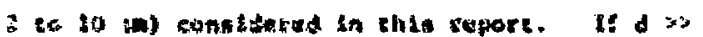

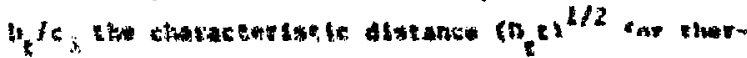

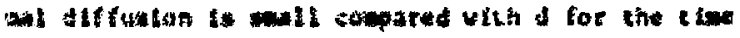

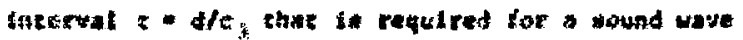

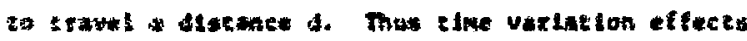

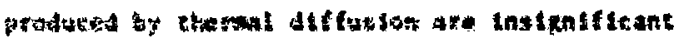

ruppared wilh ordinaty cfac variation effecto In an vast le vast. The problem of a acrege pulse noving tate a sphertcal whul 2 wose inner radius ard thichnewa sre large compared with elie wavelength of the geress putwe is ussanchally the ane problet for an atsate half space.

bst of cuupled dynanlc chermolelasticity theory

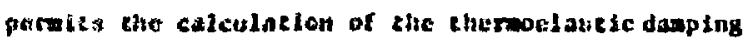
(Incernat friction) of the seress pulse at it erovels chrugh the half apace. $23-23$ ine axime value of the lox decrement is uppeximately equal to the cuuplinx constant (Ref. 22, page 393; Ref. 23, prge 501). The themotastic coupling constant for plane

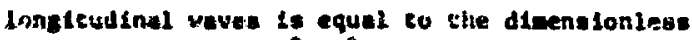

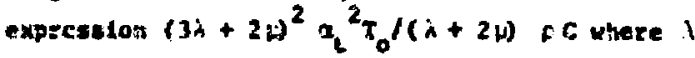
and wate the len elaste constants, $T_{0}$ is the 
reference tomperature, o is the deasity, $C$ is she sperific heat, and $a_{t}$ is the linear coeffictent of theral uxpansioll. For nfoblun the coupling constant is equal to 0.02 . Herce the aximen possible value of the $\log$ decremert is oniy 0.02 and this dappleg constant is atlatned only for stress pulses of uvelength that are orders of agnitide analier than those consideres in this report.

Let $x$ represent diatance measured fros the surface of thiz elastic haif space in a direction perpendtcular to surface of the half space. Suppose the surfact: layer $(0 \leq x \leq d)$ lo heated suddenly at t towe $t=0$ and 1 ts temperaturc 18 Intreased by the awount $\Delta T$. The aterial in the surface layer initially will not have tioc to expand. With1n the surface layer, but not on 1ts boundarses (that 1s, at $x=0$ and at $x=d$ ), the stress $\sigma_{x x}$ that acts normily to any plane parallel to the helf space surface aust equnl at $t=0$

$$
\sigma_{x x}=-\sigma_{0} \equiv-x_{t}(3 \lambda+2 \mu) \Delta T \text {. }
$$

In the intertor of the half space $\sigma_{2 x}=0$. At the surface of the half space $(x-0)$ the traction aust vanlsh and thus $o_{x x}=0$. At the boundary between the heated surface layer and the unheated interior $(x=d)$ the stress $o_{y x}=-o_{0} / 2$. Only if $o_{z x}$ has this latter value will the compressive stress pulae that orfiginates at this bounciary and travels in a pasitive $x$ direction produce an elastic displacesant at $x$ = d that is Identical to the elastic diaplacewent produced in the tensile stress mise that also criginate at this boundary at the sase time and travela in the iegative $x$ dfrection. See Eqs. (A-3) and $(A-4)$.

A tensile stress wave starts gt: the free surface at $t=0$. The elastic displacement $u_{x}$ of th is wave is given by (and obviously satisfies the wave equation $\left.\partial^{2} u_{x} / \partial x^{2}-c_{\lambda}^{-2} \partial^{2} u_{x} / \partial t^{2}\right)$

$$
u_{x:}=\left[\sigma_{0} /(\lambda+2 \mu)\right]\left(x-c_{\lambda} t\right)
$$

for $x-c_{\lambda} t<0$ and $u_{x}=0$ for $x-c_{\lambda} t>0$. The stress in this tensile wave is constant and is equal to $\sigma_{x x}=(\lambda+2 \mu)\left(\partial u_{x} / \partial x\right)=\sigma_{0} \quad A$ compressive wave starts at $t=0$ from the boundary $x=d$ and moves In the positive direction. Its stress is constant and is equal to $\sigma_{z x}=-0_{0} / 2$. The elast de displacto went in this uave is given by

$$
u_{x}=-\left[\sigma_{0} / 2(A+2, A)\left(x-d-c_{j} l\right)(A-3)\right.
$$

tor $x-d-c_{\lambda} t<0$ and $x>d$. For $x-d-c_{\lambda} t>0$ the $d i s-$ placement $u_{x}=0$. A tensile wave also fiterts at $t-0$ at $x=d$ and moves in the nagazive $x$ diraction. The stress in this wave 18 conscant and 16 equad to $\sigma_{x x}=\sigma_{0} / 2$. The elastic displacement is equal to

$$
u_{x}=\left[0_{0} / 2(\lambda+2 \mu) !\left(x-c+c_{\lambda} t\right) \quad(\lambda-4)\right.
$$

for $x-d+c_{\lambda} t=0$ and $x<d$. For $x-d+c_{\lambda} t<0$ the disflecenent $u_{n}=0$. The tenctle wave stven by Eq. $(A-4)$ reaches the free surface at $t$ ine $:=d / c$, and is reflected as a compressive wave of constant stress $\sigma_{x x}=-\sigma_{0} / 2$. This reflected wave travels in this positivi: direction and has the elastio dispiacesent (when $t>d / c_{\lambda}$ )

$$
u_{x}=-\left[0_{0} / 2(\lambda+2 j)\right]\left(x+d-c_{\lambda} t\right) \quad(i-5)
$$

for $x+d-c_{\lambda} t<0$ and $u_{x}=0$ for $x+d-c_{\lambda} t>0$. At a the $t$ that $1_{3}$ large compared witis the expression $2 \mathrm{~d} / \mathrm{c}_{\lambda}$ the total stress $o_{x \text {; }}$ in shis ineated surface layer is equal to zero because tine stress 5 . the tensile waje given by $E q .(A-1)$ exactly cancels the therasl stress given by $E_{q} .(A-i)$ and the tensilc stress in the wave given by Eq. $(A-4)$ d. canceled by the conpressive atress in the reflected wave give:s by Eq. $(A-5)$. In the region $d \leq x<\left(\left(t / c_{\lambda}\right)-2 d\right)$ the stress $\sigma_{x x}$ dlso is equal to zero because the stress of the tenstle wave given by Eq. (A-2) Is anceled by the aum of the strusses lin the conpressive itress waves glven by Eqs. (A-3) and $(A-5)$. In the region $\left[\left(t / c_{\lambda}\right)-d<x<\left(t / c_{\lambda}\right)\right]$ a compressive seress $\sigma_{x x}$ $-\sigma_{0} / 2$ exists and is followed in the region $\left(t / t t_{\lambda}\right)$ $-2 d]<x<\left[\left(t / c_{\lambda}\right)-\right]$ by a censtle stress $a_{x x}=0_{0} i 2$. Thus the traveling stress wave pruducte by the sudden heating consists of an initial conpreasive seress pulse of length of followed by a censtla -..ss pulse of length d.

\section{REFERENCES}

1. I. Frani. D. Friewnld, i. Merson, and J. Devaney, "A Laser Fusion keactor Concept Jillizing Magnetic Fields for Cavity Wall Protection," First 
Toptcal Meeting on the Technology of Controlled Nuclear Fusion (American Nuclear Soctety) April 16-18, 1974, San DHego, Calffornta (In press).

2. J. Williams, T. Merson, F. Finch, F. Schllling, and T. Frank, "A Coniceptual Laser Controlled Thermonuclear Reactor Power Plant," First TopIcal Meeting on the Technology of Controlled Nuclear Fusion (American Nuclear Soclety) April 16-18, 1974. San Diego, Callfornla (in press).

3. J. Nuckolls, J. Emett, ard L. Wood, "LaserInduced Thermonuclear Fuston," Physics Today, pp. 46-53. (August 1973).

4. F. T. Finch, E. A. Kern, and J. M. Williams, "Lager Fusion Power Plant System Analysis," First Topical Meeting on the Technology of Controlled Nuclear Fusion (American Nuclear Society) April 16-18, 1974, San Diego, Calif. (In press).

5. 5. M. W11l fams and T. G. Frank, "LaserControlled Thermonuclear-Reacter Materfals Requirements," Nu. Tech. 22, 360 (1974).

6. T. Frank, D. Dudzlak, and E. Heck, "Some Neucronics Aspects of Laser-Fusion Reactors," Pirst Toplcal Meeting on the Technology of Controlled Nuclear Fusion (American Nuclear Soclety) April 16-18, 1974, San Dlego, Calif. (in press).

7. L. A. Booth (compiler), "Central Statlon Power Generation by Laser-Driven Fusion," Los Alanos fcientific Laboratory report LA-4858-MS, Vol. 1 (February 1972).

8. H. Bethe and J. Ashkin, "Passage of Radiations Through Matter," in Experfmental Nuclear Physlcs, E. Segre, Ed., (John Wiley, New York, 1953) D. 222.

9. R. Axford, University of Illinols, personal communlcation; August 1974.

10. D. A. Frlewald, "Approximate Spherical Blast Theory and Jaser-Initiated Pellet Microexplosions," Los Alamos Scientific Laboratory report LA-5641-MS (Jure 1974).

11. M. Guinan, "Shock Wave Interactions Arising from Nen: Surface Displacement Cascades," Proceedings of Surface Ef fects in Controlled Thermonuclear Duzvices and Reactors, Argontle National Laboratory January 10-12, 1974 (in press).
12. J. Weertman, "Theory of Irradiation Growth of Fatigue Cracks," submitted to Jnl. Nic. Mat.

13. R. J. Donahue, H. McI. Clark, P. Atanme, R. K. Kumble, and A. J. McEvily, "Crack Opening Displacement and the Rate of Fatigue Crack Growth." Int. Jn1. Fract. Mech. 8, 209 (1972).

14. A. S. Tetelman and A. J. McEvily, Jr., Fracture of Structural Materials, (John Wiley, Niew York, 1967), Pp. 369-372.

15. T. E. THetz and J. W. Wilson, Behavior and Properties of Refractory Metals, (Stanford University Press, 1965), p. 112.

16. E. G. Zukas, Los Alamos Scientific Laboratory, personal communication, August 1974.

17. W. P. Mason, Physical Acoustics and the ProForties of Solids, (Van Nostrand, New York, 1958), P. 176 .

18. P. Shahinian, H. E. Watson, and H. H. Srith, "Effect of Neutron Irradiation on Fatigue Crack Propagation in Types 304 and 326 Stalnless Steels at H1gh Temperature," in Effects of Radiation on Substructure and Mechanical Properties of Metals and A1loys, ASTM STP 529. AmerIcan Soc. Test. and Mat., Philadelphta, 1973, p. 493.

19. J. Weertman, "Theory of High Temperature Intercrystalline Fracture under Static or Cyclfc Fat1gue Loads, With or Without Irradiation Damage," Met. Traas. 5, 1943 (1974).

20. G. L. Miller, Tantalum and Nioblum, Academic Press, New York, 1959), p. 426.

21. B. A. Boley and J. H. Weiner, Theory of Thermal Stresses, (John Wiley, Nev York, 1960).

22. J. D. Acilenbach, Wave Propagation in :1autic Solids, (North-Holland, Amsterdam, 1973).

23. A. S. Nowick and B. S. Berry, Anelagtic Relaxation in Crystalline Solide, (Academic Press, New York, 1972). 